\title{
Implementation of Beam Steering using Phased Array Antennas
}

\author{
Jestin John Mathew, Nitish HS, Dr. Jayavrinda V, Dr. Raghunandan S \\ Nitte Meenakshi Institute of Technology, Karnataka, Bangalore-560064 ,India
}

\begin{abstract}
Beam steering is a phenomenon of varying the direction of the main lobe in the radiation pattern, so in radar systems it can be achieved by changing the relative phases of the antenna being implemented.But,some of the setbacks of implementing beam steering in mechanical antennas like microstrip antenna is that the directivity and, gain is low, and interference is very high.Whereas,in our project we have implemented beam steering using phased array antennas which has produced better results.So,an phased array antenna is electronically scanned antenna ,which produces a beam of radio waves that can be steered to different points based on our requirements by keeping the antenna stationary.Also,these type of antennas require less maintenance as compared to mechanical antennas.
\end{abstract}

Keywords:- Phased array antennas, Beam steering, Directivity, Interference.

\section{INTRODUCTION}

Generally,a basic antenna like an microstrip antenna has a lot of transmission losses, as the directivity is not very high ,this is because the side lobes are big and the main lobe is also of a similar size as that of the side lobes, and a lot of energy is wasted.Also,the property of beamsteering cannot be implemented effectively in a basic antenna.But,by using a phased array antenna we can achieve beamsteering effectively and the energy losses can be minimised greatly,as the main lobe size is increased greatly ,by this energy transmitted along with the directivity can be increased.

\section{A. Phased Array Antenna}

A phased array is basically an electronically scanned array, it works in such a way that it creates a beam of radio waves which can be steered to point in different directions by keeping the antenna stationary,hence it can also be called as computer-controlled array of antennas.Normally,in the phased array antenna the radio frequency current from the transmitter is fed to the the individual array elements of antenna based on the perfect phase relationship ,by this radio waves from all the array elements of antenna sum up together to increase the radiation in the desired direction, while cancelling each other to reduce radiation in the undesired direction.In a phased array antenna, the power from the transmitter is fed to the antennas through devices called as phase shifters, these are primarily controlled by a computer system, thus it can alter the phase electronically, which results in steering the beam of radio waves to different directions. A phased array antenna should consist of many array of small antennas ,it can sometimes even be in thousands to attain high gain, usually phased arrays are mainly practical at the high frequency end of the radio spectrum, whereas its observed that in UHF(Ultra High Frequency) and microwave bands, the antenna elements are conveniently small.

The principle of this antenna is based on the effect of interference, i.e. a phase-dependent superposition of two or more radiation sources. It can be observed that the in-phase signals amplify each other, while counter-phase signals cancel each other out. When two radiators emit a signal in the same phase shift, a superposition is achieved - the signal is amplified in the main direction and attenuated in the secondary directions.Therefore,to attain high directivity, many radiators are implemented in the antenna field.



Fig 1:- Phased Array Antenna

\section{B. Block Diagram}

Initially , all the required simulation parameters like frequency,wavelength,time period,spatial discretization, etc. are declared.In this project,we have used the phased array antenna which is composed of array of 7 point sources,these are uniformly spaced in a linear form,this is basically called as uniform linear array (ULA). The separation between the antennas is denoted by the parameter $\mathrm{d}$. When the separation is smaller, the directivity of the array is narrower. Each and every antenna element in the array is fed with a relative phase shift of "delta" with respect to the adjoining one (the rightmost antenna is called the reference antenna, in this no phase shift is applied, i.e. delta $=0$ ).So,as the phase shift (delta) is swept, the array 
patterns also change accordingly. So, if the delta is less than or equal to 360 degree ,the electric field with respect to angle for each individual antenna is calculated and based on it the antenna radiation pattern is animated.Further,again the delta value is incremented and the same process is followed till the delta exceeds 360 degree,if it exceeds then the process stops.

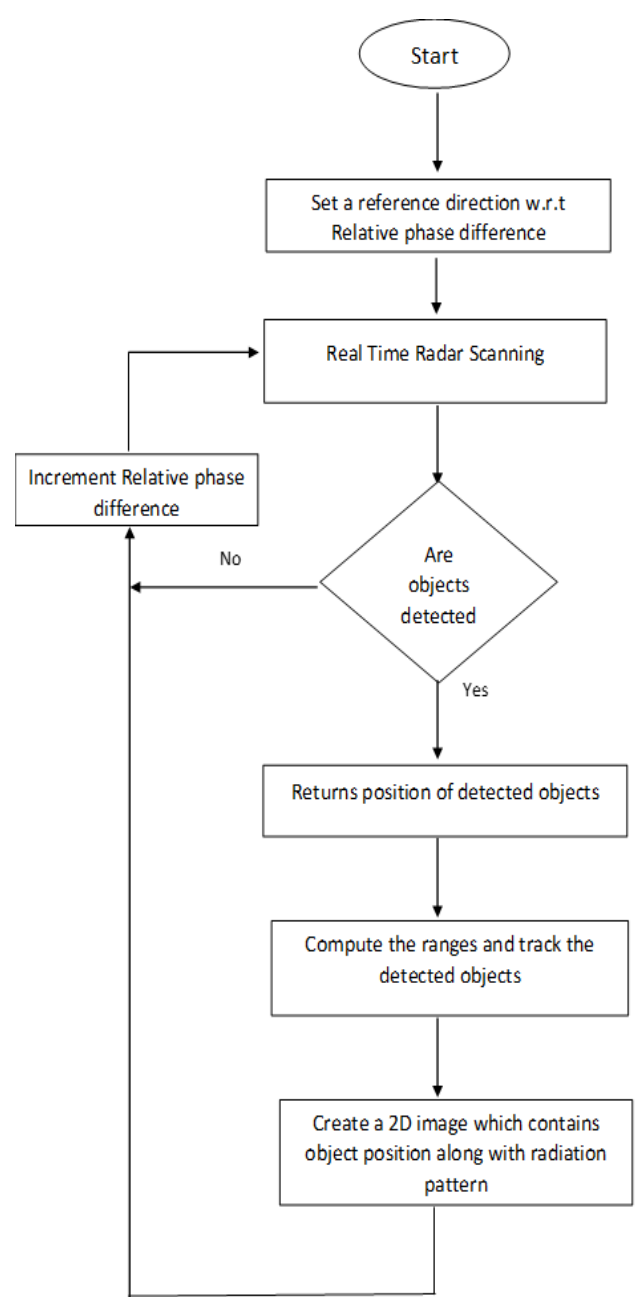

Fig 2:- Block Diagram of the process of beam steering using phased array antennas.

\section{RESULTS}

As mentioned earlier,we are using phased array antennas to implement beam steering in this project.This antenna consists of an array of 7 point sources,these are uniformly spaced in a linear form, hence this is called a uniform linear array (ULA). The separation between the antennas i.e arrays is denoted by the parameter $\mathrm{d}$. When the separation is smaller, the directivity of the array also gets narrower. Each and every antenna element in the array is fed with a relative phase shift of "delta" with respect to the adjoining one (the rightmost antenna is called the reference antenna, in this no phase shift is applied, i.e. delta=0). So, as the phase shift (delta) is swept, the array patterns also change accordingly.From this, we were able to observe beam steering of the antenna from 0 degrees to 360 degrees incrementally.It can also be absorbed from the radiation pattern that there's an increase in size of the main lobe on comparison with the side lobes.

Figure 3 and figure 4 shows the radiation pattern of the antenna at delta values of 90 and 270 degrees respectively.

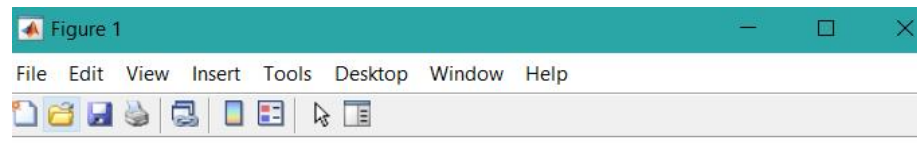

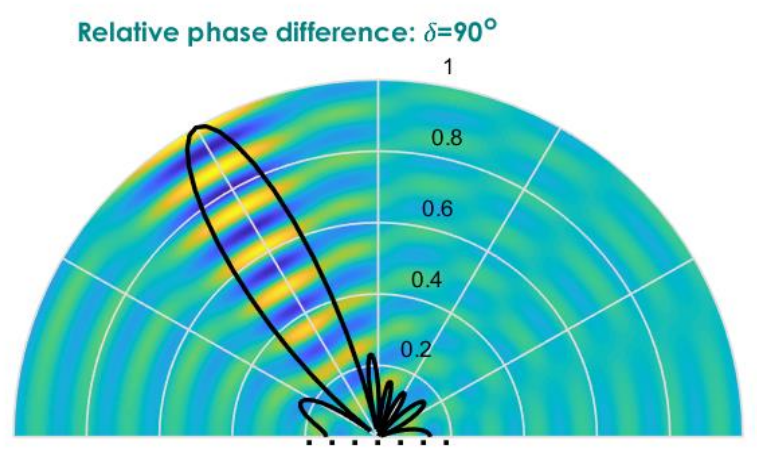

Antenna separation: $d=\lambda / 2$

Fig 3:- Radiation pattern at a delta value of 90 degree

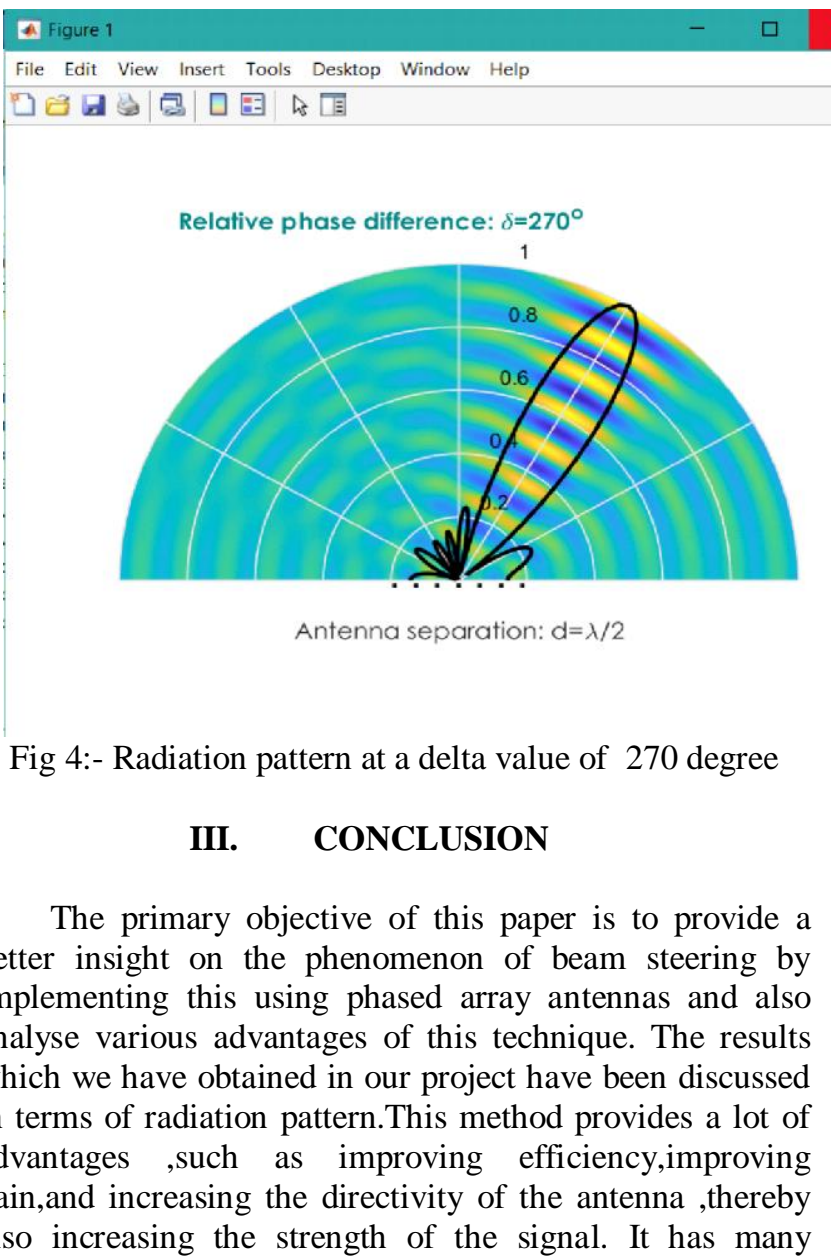


applications in the form of surveillance and tracking , this is because the transmitted beam can be steered effectively in different directions within a few milliseconds.Also,phased array provides an free eligible Dwell time as well as multi function operations by emitting numerous beams simultaneously.Beam steering antennas allow graceful network degradation to occur through network reconfiguration, continued functionality of the unaffected regions of the network, a degree of network recovery by resource reallocation which acts as a backbone once the connections are lost.One of the limitations of this technique is that it's expensive to implement. Research on beam steerable antennas is getting a good amount of interest. And, we were able to successfully implement beam steering technique using phased array antennas and also get the desired results from the simulation.

\section{REFERENCES}

[1]. G. Hakobyan and B. Yang, "High-Performance Automotive Radar: A Review of Signal Processing Algorithms and Modulation Schemes", IEEE Signal Processing Magazine, vol. 36, no. 5, pp. 32-44, Sept. 2019.

[2]. Zivin Park, Jenshan Lin "A Beam-steering Microstrip Antenna for Non-Contact Vital Sign Detection," IEEE Antennas and Wireless Propagation Letters, vol. 10, pp. 235-238, 2011

[3]. Y. Yusuf and X. Gong, "A patch antenna with low cost phased array with analog beam steering using refractive coupling and mutual loading," IEEE Antennas Wireless Propag. Lett., vol. 7, pp. 81-84, 2010

[4]. Iyemeh Uchendu and James Kelly "Survey of Beam Steering Techniques Available for millimeter wave application" Progress in Electromagnetics Research B, vol. 68, 35-54, 2016.

[5]. Taekso Ji, Hargsoon Yon, J. K .Abraham, Vijay K. Varadan," A Ku-Band antenna array feed distribution network with ferroelectric phase shifters on Silicon," IEEE Transactions on microwave Theory and Techniques, vol 54, no. 3, 2006.

[6]. M. K. Saleem, H. Vettikaladi, M. A. S. Alkanhal and M. Himdi, "Lens Antenna for Wide Angle Beam Scanning at $79 \mathrm{GHz}$ for Automotive Short Range Radar Applications", IEEE Transactions on Antennas and Propagation, vol. 65, no. 4, pp. 2041-2046, April 2017.

[7]. D. R. Jackson, and N. G. Alexopoulos, "Gain increase methods for printed circuit antennas," IEEE Trans. Antennas Propagation, vol. 33, no. 9, pp. 976-987, 1985.

[8]. Abel Abdul Zandamela, Korbinian Schraml1,Vasan Jantarachote, Suramate Chalermwisutkul, Dirk Heberling,Max J. Ammann, and Adam Narbudowicz, "On the Efficiency of Miniaturized $360^{\circ}$ BeamScanning Antenna ",EuCAP - European Conference on Antennas and Propagation, London, UK, 2019.
[9]. H. Kirino,and K Ogawa,"Various applications of lowcost phased array antennas with mechanical phase shifters",IEEE-APS Topical Conference on Antennas and Propagation in wireless communications, 2014.

[10]. H. Attia, L. Yousefi, M. M. Bait-Suwailam, M. Said Boybay and O. M. Ramahi, "Analytical model for calculating the radiation field of Micro strip antenna with artificial magnetic superstrates.Theory and Experiment," IEEE Transactions on Antennas and Propagation, vol. 59, no 5, pp.1438-1445,2011.

[11]. H. Griguer, M. Drissi, E. Marzolf "Design and characterization of a tunable DNG superstrate for relatively small beam steering antennas," Proceeding of META'10, the 2nd International Conference on Metamaterials, Photonic Crystals and Plasmonics, pp. 255-259, Cairo, Egypt, February 2010.

[12]. R. Abou-Jaoude and M. Grace, "Test systems for automotive radar," VTC2000-Spring. 2000 IEEE 51st Vehicular Technology Conference Proceedings (Cat. No.00CH37026), Tokyo, Japan, 2000, pp. 492-495 vol.1. 